\title{
Improving the mood of elderly with coloured lighting
}

\author{
Andre Kuijsters ${ }^{1}$, Judith Redi ${ }^{1}$, Boris de Ruyter ${ }^{2}$, and Ingrid \\ Heynderickx ${ }^{1,2}$ \\ ${ }^{1}$ Delft University of Technology, Delft, The Netherlands \\ ${ }^{2}$ Philips Research, Eindhoven, The Netherlands
}

\begin{abstract}
The ACE project aims at developing an adaptive ambience creation platform which can improve the well-being of elderly people in care centers. The system will contain a sensing platform, which measures and analyses the context in a room and an ambience creation platform, which creates ambiences with proven effectiveness for improving the mood of the elderly. It has been shown in literature that lighting can influence people's mood; however, consistent effects have scarcely been documented. The effects of lighting characteristics on atmosphere perception, on the other hand, are better documented. It has been proved that ambiences with a positive affective meaning (e.g. cosy, activating) can be created with the combination of functional and atmospheric LED lighting. We expect that these ambiences can improve the mood of elderly towards its affective meaning. The experiments we intend to perform in order to achieve that goal are discussed in the paper.
\end{abstract}

Keywords: Mood, Ambience, Atmosphere perception, LED lighting, Elderly

\section{Introduction}

The advent of LED technology radically increased the possibilities for generating light of various colours and intensities at any place and time. As a consequence, the variety of new opportunities for designing lighting in a room has grown tremendously. These new opportunities do not only concern functional lighting effects, but enable also a richness of decorative and ambient lighting effects. A combination of these effects can be used to more easily take into account the needs of occupants in a room, and possibly to improve his/her well-being. It has been shown that the combination of functional and decorative ambient lighting can be used to create ambiences with a clear affective meaning [1]. How people experience the affective meaning of these ambiences is referred to as atmosphere perception. In addition, it is known that colour can affect people's mood [2]. Hence, we believe that also (coloured) lighting ambiences have the potential to improve the mood and emotional state of the people occupying a room.

Our idea is based on biological and psychological processes that, triggered by light and colour, impact on mood. The biological effects of bright light on melatonin levels are well documented $[3,4]$. Night time melatonin release can be phase advanced or delayed with the right exposure to bright light [4], thereby influencing the circadian rhythm of humans. Disruptions to circadian rhythms (e.g. due to jetlag or shift work) 
can cause mood disorders [5]. Seasonal affective disorders (characterized by depression symptoms in the winter) are also considered to be the result of a disturbance of the circadian rhythm, caused by the failure to adapt to the shift in day length as a result of seasonal change. Bright light therapy, existing of 1500lx or more at eye level, significantly reduce depression symptoms for people with seasonal mood disorders [6].

The psychological effects of white light on mood have been studied extensively, revealing mixed results. Some studies reveal no significant effects of white lighting on mood [7]. Other studies reported only small interaction effects between gender, illumination and colour temperature [8-11]. For instance, Knez and colleagues [11] reported that females' negative mood decreased while working under warm light conditions $(3000 \mathrm{~K})$ and increased under cool light conditions $(4000 \mathrm{~K})$, while an opposite effect was found for males. However, in a later study Knez and Enmarker [10] found an opposite result; females' negative mood increased more under cool white light conditions (4000K), while men's negative mood increased more in warm white light conditions $(3000 \mathrm{~K})$.

Studies on the psychological effects of colour (e.g. coloured walls) on mood suggest that the right amount of ambient colour can improve the mood of people. Küller and colleagues [2] found that office workers which judged their offices as colourful experienced a better mood throughout the year than office workers that judged their offices as neutral or colourless.

This short summary of the existing literature clearly shows that lighting ambiences and colour can indeed impact people's mood. So far, the effects of these two elements on mood have been studied disjoint. The advent of LED-based systems drastically increased the possibilities of combining functional and coloured lighting, e.g. creating different colours on walls. Therefore, new opportunities in influencing affective states with colour and light are now open. Based on the literature, we hypothesize that such a combination of functional and coloured lighting is beneficial to mood improvement, and we aim at extending the existing research in order to better understand the direction and extent of such improvement.

A successful design of mood-improving ambiences based on lighting is expected to be of high relevance for different applications, such as in classrooms, hospital rooms, and day care facilities. Our research focuses on care centres for elderly people, where negative mood states are often experienced, especially at the moment of relocation. The goal of our project is therefore to design an adaptive ambience creation system with proven effectiveness in improving negative moods of elderly, e.g. relaxing those elderly that feel disoriented and anxious, or activating those who feel sad and depressed.

\section{ACE Project}

The Adaptive Ambience Creation in Care Centres for Elderly project (ACE) aims at designing a system that can automatically adapt the ambience in a care centre room to the needs of the elderly occupying it. A number of factors have to be considered to create the appropriate ambience, among which are the context in the room, the intended ambience in the room, the activities and mood of the inhabitants and their personal preferences. Thus, a sensing platform that measures and analyses the context in the room and the activities and mood of its inhabitants is needed to enable the 
ambience creation platform to generate the appropriate ambience. The sensing platform will be designed using off-the-shelf low-cost sensors and intelligent algorithms. The affective state of the room's occupant will be determined by analysing e.g. posture, type and level of activity, speech characteristics and facial expressions. The output of the sensing platform should be the indication for a desired ambience change and its desired affective direction.

The ambience creation platform will be based on a combination of functional and atmospheric light, to which sound (i.e. music) and fragrance could be added in a second stage. As mentioned before, the platform will at first focus on two ambiences, with an associate relaxing and activating atmospheres respectively. How to create these ambiences for elderly needs more research. For younger people there is already more insight on how to combine atmospheric light with functional light in order to convey specific atmospheres $[12,13]$ (see also chapter 3). It is also known that there is a considerable degree of freedom in the actual lighting settings before a given atmosphere is lost. Hopefully, this degree of freedom also exists for elderly people, and may then be used to personalize the system towards optimal or preferred performance for individual elderly. In that case, the elderly is expected to tune some of the light characteristics that determine a given atmosphere once, and the resulting settings are then stored in the ambience creation system.

Near the end of the project, the sensing and ambience creation platforms will be integrated, using a control unit, into a complete intelligent ambience creation system. The system should be able to automatically detect the atmosphere and context in a room and the emotional state of its inhabitant. Based on the outcome of the sensing platform in combination with information on the intended ambience and personal preferences of the elderly, the appropriate ambience will be created. The control of the platform and the ability to include personal preferences are expected to be crucial for the acceptance of the platform.

\section{Mood and atmosphere perception}

At the core of the implementation of the ambience creation platform is the investigation of the added value of LED technology in improving the mood of elderly. We base our approach on the notion of atmosphere [14]. Atmosphere differs from mood; it does not represent the affective state of a person, but rather the affective state of an environment. As such, an atmosphere may have the potency to changing a person's mood. Independent of a person's emotional state when entering a room with a specific atmosphere, the affective state of the environment may be immediately recognized. On the longer term the atmosphere is expected to change the emotional state of the person towards that same affective state. The latter is supported by the underlying aspects of mood and atmosphere perception, which suggest a relation between the two concepts. Mood can be quantified in a two-dimensional affective space, with the bipolar axes pleasantness and arousal [15]. Atmosphere can be quantified in a four-dimensional affective space, of which the first three dimensions, i.e. coziness, liveliness and tenseness, are most important [14]. Clearly the dimensions of coziness and tenseness in atmosphere perception have a link with the pleasantness dimension of mood. The same holds for the dimension of liveliness in atmosphere perception and the dimension arousal in mood. For instance, being excited is a high arousing, pleasant mood state, while an activating ambience is a high arousing (i.e. 
lively), pleasant atmosphere (see Figure 1). Hence, because of this underlying relationship we believe that ambiences with a recognized, positive atmosphere could to some extent drive the occupant's mood towards the same affective direction.

Recent research revealed consistent effects of lighting characteristics on atmosphere perception. Warm white light $(2800 \mathrm{~K})$ was perceived as more cozy and less tense as compared to cold white light (6000K) [14]. Increasing the illuminance (from 40 to 4001x) resulted in a less tense, and more lively perception of the ambience [14]. Spot light was considered livelier and less tense than more diffuse light. Finally, yellow and red colours at low saturation were found to contribute to coziness perception, while highly saturated red light was considered as tense [16].

Thus, the knowledge on atmosphere perception of a younger population is substantial, but so far there is no information on how elderly people perceive these light effects. Hence, by first investigating the atmosphere perception of elderly, we expect to be more effective in indentifying the lighting components that impact the mood of elderly people.

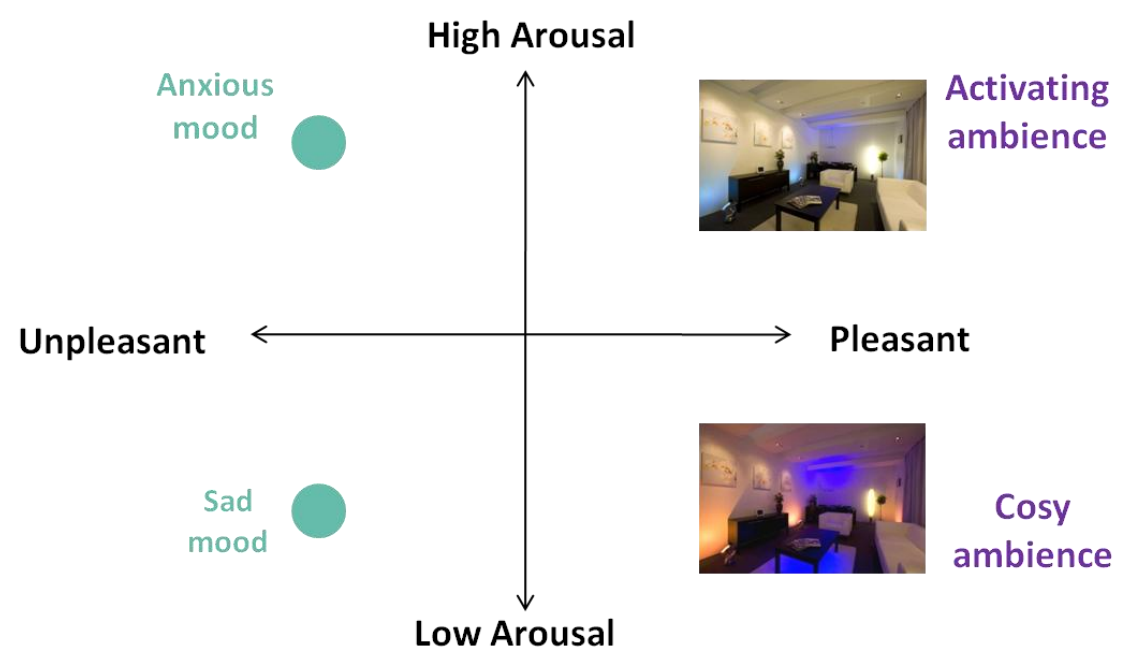

Fig. 1. Two-dimensional affective space [15]. Containing two unpleasant mood states, low arousing (Sadness) and high arousing (Anxiousness) and two pleasant affective ambiences, low arousing (Cosy) and high arousing (Activating).

\section{Plan of research}

The first question is whether elderly people perceive atmosphere in a way comparable to that of younger people. A positive answer in this sense would make previous knowledge on the affective meaning of ambiences $[1,8]$ exploitable for the purposes of our research. It is known that younger people can recognize and distinguish between ambiences created with lighting [1]. We will investigate whether age related deterioration of the visual system and possible different attitudes towards coloured lighting influence the atmosphere perception. The goal of the experiment is to identify 
a high arousing, positive ambience (i.e. activating) and a low arousing, positive ambience (i.e. cosy).

A second and core question is whether the identified ambiences can really induce a mood change in accordance with their affective meaning. In other words, an activating ambience should be more effective in activating sad elderly (i.e. increasing both their arousal level and pleasure) than a neutral ambience (see Figure 2). On the other hand, a cosy ambience should be more effective in relaxing anxious elderly (i.e. decreasing their arousal level and increasing their pleasure) than a neutral ambience (see Figure 3).
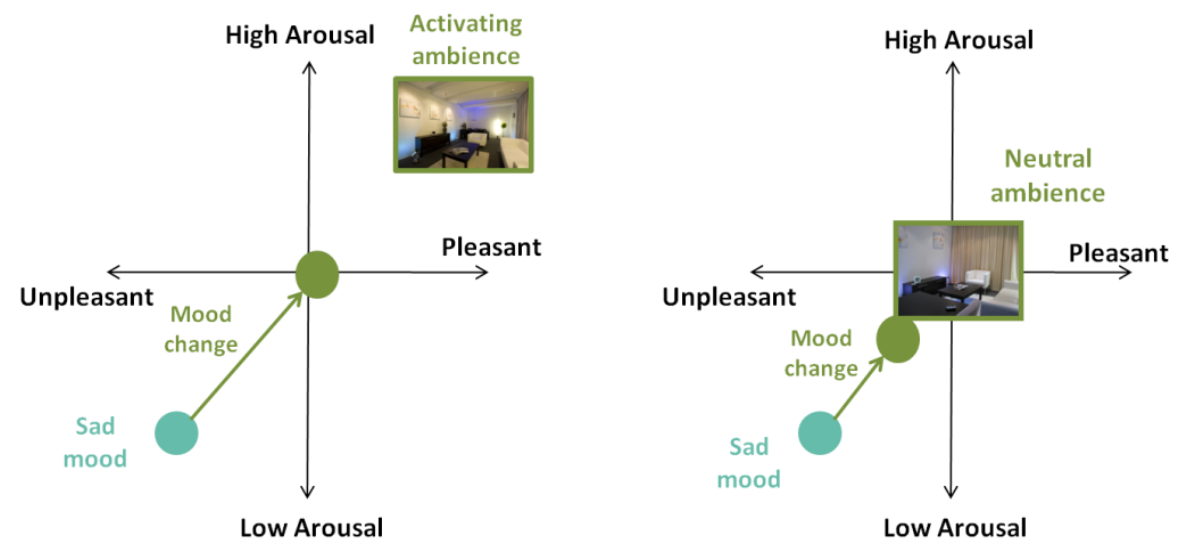

Fig. 2. Expected influence of an activating ambience (left) and a neutral ambience (right) on an anxious mood state; depicted in the two-dimensional affective space [15].
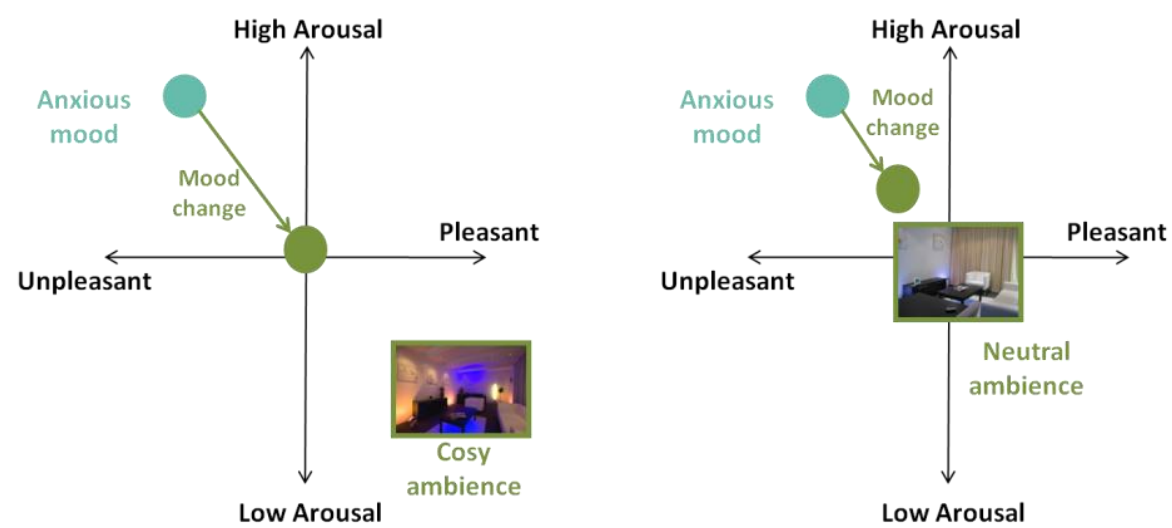

Fig. 3. Expected influence of a cosy ambience (left) and a neutral ambience (right) on an anxious mood state; depicted in the two-dimensional affective space [15].

We address the core question in a two step approach. In the first step, the effect of the ambiences on the mood of the elderly will be investigated under controlled laboratory conditions. In this way we can control all other possible influences (e.g. temperature, 
sunlight) on the mood of the elderly. However, the experienced mood states of the elderly participating in the experiment are most likely different from the mood states (i.e. sadness and anxiousness) we aim to improve. Therefore we will induce negative mood states. Different mood induction procedures exits for inducing mood states in the laboratory (see [17] for a review), and include listening to music, viewing movies and viewing affective slides. The induction should be effective in inducing the targeted mood and the mood state should last after the induction procedure. Changes in the mood of elderly will be measured both subjectively and objectively. The elderly will assess their current mood state on several times during the experiment on the pictorial Self Assessment Manikin (SAM). The heart rate, skin conductance and skin temperature of the elderly will be monitored throughout the whole experiment. In a later stage, the effect of the affective ambiences will be investigated under more realistic circumstances. In collaboration with the Vitalis WoonZorg Groep Eindhoven, the system will be tested with elderly experiencing actual negative mood states.
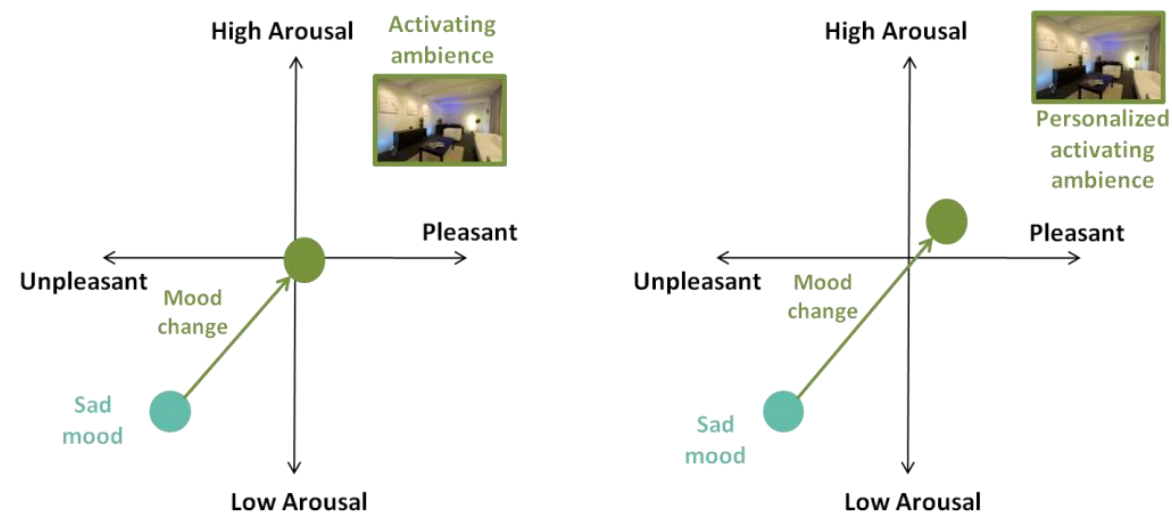

Fig. 4. Expected influence of an activating ambience (left) and a personalized activating ambience (right) on a sad mood state; depicted in the two-dimensional affective space [15].

The third question is test the effect of personalization of the ambiences. It is known that quite some variation in lighting settings is allowed within an ambience, without losing its affective meaning [13]. This variation may be used by people to personalize their ambiences; i.e. to make the cosy ambience cosier and the activating ambience more activating according to their own perception. The elderly participants will be able to adjust several lighting setting (e.g. colour in the ambience, illumination, colour temperature of the white light), starting from the original ambiances (e.g. cosy or activating). The resulting variation in settings will illustrate how important personalization is for the elderly. Subsequently, the impact of a personalized ambience will be compared to the impact of an averaged ambience (as illustrated in Figure 4). In a final stage other adaptive senses (e.g. music and fragrance) might be added to enhance the affective experience of the ambience.

The ACE project is expected to improve the well-being of elderly people in care centres, which is, despite the tendency of a longer independence for elderly, still a growing group of people in the future. Improving the well-being of these elderly is expected to reduce their demand on the nursing personnel. In addition, the basic ideas 
resulting from this project can be reused in different contexts, such as hospital rooms and schools.

Acknowledgments. The ACE project is a collaboration between Philips and TUD funded by PointOne of Agentschap NL. We are grateful to Vitalis WoonZorg Groep for supporting us in our research.

\section{References}

1. Vogels, I.M.L.C., de Vries, M., van Erp, T.A.M.: Effect of Coloured Light on Atmosphere Perception. In: Association Internationale de la Couleur (AIC). (2008)

2. Küller, R., Ballal, S., Laike, T., Mikellides, B., Tonello, G.: The impact of light and colour on psychological mood: a cross-cultural study of indoor work environments. Ergonomics 49, 1496--1507 (2006)

3. Mclntyre, I.M., Norman, T.R., Burrows, G.D., Armstrong, S.M.: Human melatonin suppression by light is intensity dependent. Journal of Pineal. Research 6, 149--156 (1989)

4. Lewy, A., Wehr, T., Goodwin, F., Newsome, D., Markey, S.: Light suppresses melatonin secretion in humans. Science 210, 1267--1269 (1980)

5. Boyce, P., Barriball, E.: Circadian rhythms and depression. Aust Fam Physician 39, 307$310(2010)$

6. Golden, R.N., Gaynes, B.N., Ekstrom, R.D., Hamer, R.M., Jacobsen, F.M., Suppes, T., Wisner, K.L., Nemeroff, C.B.: The Efficacy of Light Therapy in the Treatment of Mood Disorders: A Review and Meta-Analysis of the Evidence. Am J Psychiatry 162, 656--662 (2005)

7. Baron, R.A., Rea, M.S., Daniels, S.G.: Effects of indoor lighting (illuminance and spectral distribution) on the performance of cognitive tasks and interpersonal behaviors: The potential mediating role of positive affect. Motivation and Emotion 16, 1--33 (1992)

8. McCloughan, C.L.B., Aspinall, P.A., Webb, R.S.: The impact of lighting on mood. Lighting Research and Technology 31, 81--88 (1999)

9. Knez, I., Niedenthal, S.: Lighting in Digital Game Worlds: Effects on Affect and Play Performance. CyberPsychology \& Behavior 11, 129--137 (2008)

10. Knez, I., Enmarker, I.: Effects of Office Lighting on Mood and Cognitive Performance And A Gender Effect in Work-xRelated Judgment. Environment and Behavior 30, 553--567 (1998)

11. Knez, I.: Effect of indoor lighting on mood and cognition. Environmental psychology 39-$51(1995)$

12. Seuntiëns, P.J.H., Vogels, I.M.L.C.: Atmosphere creation: Atmosphere and light characteristics. Philips Research (2008)

13. Choy, K.: Atmosphere creation in the living room: The freedom of light characteristics in atmosphere perception for the living room. Media and Knowledge Engineering. Delft University of Technology, Delft (2009)

14. Vogels, I.M.L.C.: Atmoshere metrics. In: Westerink, J.H.D.M., Ouwerkerk, M., Overbeek, T.J.M., Pasveer, W.F., de Ruyter, B. (eds.) Probing Experiece: From Assessment of User Emotions and Behaviour to Development of Products, vol. Philips Research Book Series. Springer, The Netherlands (2008)

15. Russell, J.A., Pratt, G.: A description of the affective quality attributed to environments. Journal of Personality and Social Psychology 38, 311--322 (1980)

16. Vogels, I.M.L.C.: How to make life more colorful: From image quality to atmosphere experience. In: 17th Color Imaging Conference, pp. 123--128. (2009)

17. Wirz-Justice, A., Benedetti, F., Berger, M., Lam, R.W., Martiny, K., Terman, M., Wu, J.C.: Chronotherapeutics (light and wake therapy) in affective disorders. Psychological Medicine: A Journal of Research in Psychiatry and the Allied Sciences 35, 939--944 (2005) 